\title{
Promoter Methylation, BRAF Mutation Analysis and Topoisomerase IIa Expression for the Detection of Endometrial Carcinoma in Liquid Based Cytology Samples
}

\author{
Aris Spathis ${ }^{1}$, Vasileios Sioulas ${ }^{2}$, Evaggelia Aga $^{1}$, Dionissios Aninos ${ }^{1}$, Christine Kottaridi ${ }^{1}$, \\ Emmanouil Terzakis $^{3}$, Charalambos Chrelias ${ }^{2}$, Petros Karakitsos ${ }^{1}$
}

${ }^{1}$ Department of Cytopathology, School of Medicine, University General Hospital “ATTIKON”, National and Kapodistrian University of Athens, Athens, Greece; ${ }^{2} 3$ rd Department of Obstetrics and Gynecology, School of Medicine, University General Hospital "ATTIKON", National and Kapodistrian University of Athens, Athens, Greece; ${ }^{3}$ 2nd Department of Gynecology, St. Savvas Anticancer-Oncologic Hospital, Athens, Greece.

Email: aspathis@med.uoa.gr

Received March 22 ${ }^{\text {nd }}, 2013$; revised April 23 ${ }^{\text {rd }}, 2013$; accepted April 30 ${ }^{\text {th }}, 2013$

Copyright (C) 2013 Aris Spathis et al. This is an open access article distributed under the Creative Commons Attribution License, which permits unrestricted use, distribution, and reproduction in any medium, provided the original work is properly cited.

\begin{abstract}
Cancer of the corpus uteri remains the most common gynecological related cancer in developed countries. Cytology, after the induction of liquid based cytology, has reemerged as a possible first line non-interventional diagnostic procedure with promising results. Apart from slide preparation for cytology diagnosis, LBC allows the application of elaborate molecular tests on the residual material. Samples from 74 symptomatic women were collected in ThinPrep ${ }^{\circledR}$ PreservCyt medium, from witch immunocytochemical and molecular tests were performed. Final diagnosis of 39 endometrioid carcinomas, 20 non-endometrioid carcinomas and 15 non-malignant was set after hysterectomy. Topoisomerase IIa expression was common (42\%) in both types of cancer. Promoter methylation analysis revealed that hMLH1 is commonly methylated in cancers $(52.7 \%)$, CDKN2A and MGMT less often $(27.1 \%)$ and RARB rarely methylated (8.4\%). BRAF activating mutation V600E was a rare event (8.4\%) only found in low grade endometrioid carcinomas. Topoisomerase IIa expression correlated with BRAF mutations, hMLH1 and to lesser extent with CDKN2A methylation. Almost none of the biomarkers were positive in cytological negative or hyperplastic without atypia samples. Detection of methylation in any gene displayed sensitivity, specificity, PPV and NPV similar to cytology of cancer. However, inclusion of cytology diagnosis of hyperlasias with atypia increased sensitivity and NPV of cytology outperforming methylation of any gene. Further evaluation of the panel of promoter methylation, especially in cytology diagnoses of hyperplasia with or without atypia should be evaluated since initial results are promising. Even though methylation of MGMT and RARB are rare events, some patients could be benefit from specific chemotherapeutics that target either of them or the more frequently expressed topoisomerase IIa.
\end{abstract}

Keywords: Endometrial Cancer; Promoter Methylation; Topoisomerase IIa; BRAF; Liquid Based Cytology

\section{Introduction}

Cancer of the corpus uteri remains the most common gynecological related type of cancer in developed countries, while it is outnumbered only by cervix uteri cancer in developing countries due to lack of screening programs [1]. The increase in endometrial carcinomas observed in developed countries has been linked to increased life expectancy, obesity and tamoxifen use $[2,3]$.

A dualistic model of endometrial cancer development has been proposed with two types of endometrial cancers with distinct histopathologic findings [4], survival rates
[5] and more recently molecular alterations that seem to be characteristic for each type [6,7] and their precursors [8-10]. Type I, which accounts for $70 \%-80 \%$ of endometrial carcinomas, include grade I and II endometrioid adenocarcinomas, that are considered to be low grade, are usually hormone-dependent and more often in pre- or peri-menopausal women [11]. The less common type II, on the other hand, includes grade III endometrioid and all non-endometrioid carcinomas (clear cell, serous, mixedtype carcinomas and carcinosarcomas), are non-hormone dependent and usually found in post-menopausal women [11]. Apart from histopathologycal and epidemiological 
differences, type II carcinomas have substantially poorer survival [5] and adjuvant chemotherapy is common, after hysterectomy $[12,13]$.

Most endometrial carcinomas are presented with abnormal uterine bleeding and less than $1 \%$ are asymptomatic [14]. Even though trans-vaginal ultrasound is the most tolerable for patients first line diagnostic test, it usually shows low positive predictive value [15]. Pippele biopsy poses an alternative test that is less tolerable and targets a specific area $[15,16]$, while hysteroscopy and curettage is the least tolerable option that requires patient admition. Abnormal endometrial cells in Pap smears are a circumstantial finding, while blood and overlapping cells hamper safe cytology diagnosis [17]. Liquid based cytology and endometrial sampling brushing have resurfaced the use of cytology in identifying endometrial lesions [18-22].

Our aim was to combine, via a single sampling method, cytologic and molecular testing results in order to further enhance diagnostic efficacy of liquid based cytology endometrial brushings. A panel of genes commonly methylated in cervical cancer was selected, including the tumor suppressors connected with either cell cycle regulation $\left(\mathrm{CDKN} 2 \mathrm{~A}, \mathrm{p} 16^{\mathrm{INK} 4 \mathrm{~A}}\right)$, or with epithelial differentiation (retinoic acid receptor RARB) and genes encoding DNA repair proteins as human MutL Homolog 1 (hMLH1) and O6-Methylguanine DNA methyl-transferase (MGMT) [23-26].

\section{Materials and Methods}

\subsection{Sample Collection}

A total of 74 direct endometrial cavity samplings were collected from women who were admitted to the hospitals $3^{\text {rd }}$ Department of Obstetrics and Gynecology and were scheduled to undergo diagnostic or therapeutic dilatation and curettage and/or hysterectomy. All women presented with abnormal bleeding with most having pathological thickening of the endometrium. The mean age of women was 63.1 years of age.

Samples for cytology were collected prior to any other intervention using a Y-shaped device designed for endometrial sampling (EndoGyn, Biogyn S.N.C., Mirandola, Italy). The thin two armed sheathed curette allowed collection of endometrial cells without any sample infection from cervical cells. Cells were rinsed into Cytolyt ${ }^{\mathbb{B}}$, Hologic, USA), that allows cell preservation, hemolysis and mucolysis. Cells were pelleted via centrifuging and transferred into the fixative (PreservCyt ${ }^{\circledR}$, Hologic, USA). A slide was prepared using the automated slide processor (ThinPrep ${ }^{\circledR}$ TP2000, Hologic, USA) and stained according to Papanicolaou. Cytology diagnosis was given according to the 1994 World Health Organization (WHO) classification scheme 22 with minor modifications. Therefore, the classification used in the current study was atrophic endometrium, hyperplasia without atypia (simple or complex hyperplasia), hyperplasia with atypia and positive for malignancy.

\subsection{Immunocytochemistry}

Immunocytochemical staining of Topoisomerase $\mathrm{II} \alpha$ (1:80, clone 3 F6, Leica Biosystems Newcastle Ltd., UK) was performed on a fully automated slide system (Bond$\mathrm{Max}^{\mathrm{TM}}$, Leica Biosystems, UK), using a commercial kit (Bond $^{\mathrm{TM}}$ Polymer Refine, Leica Biosystems, UK), that contains a secondary antibody linked to a HRP coupled polymer, DAB chromogen and heamatoxylin counterstain. Samples were considered positive if nuclear staining for Topoisomerase II $\alpha$ was identified.

\subsection{Methylation Specific PCR-MethyLight Analysis}

Methylation specific PCR for hMLH1, CDKN2A and MGMT was performed as previously described using a commercial kit (Amplicolon, Bird Srl, Italy) [24]. Bisulfite converted DNA was amplified with primers specific for either the unmethylated or the methylated form in separate reactions. PCR products were analyzed on $2 \%$ agarose gels for presence of either form. If a PCR product was detected only in the unmethylated reaction, the sample was characterized as unmethylated, while presence of a PCR product in the methylated reaction characterized the sample as methylated, regardless of the result of the unmethylated reaction. Absence of a product from both reactions characterized the sample as invalid. Both unmethylated and methylated DNA controls were included in the kit.

For RARB two sets of primers and Taqman probes were designed using Beacon Designer (PremierBiosoft, USA). Real-Time PCR amplification for RARB promoter methylation detection was performed using a FAM labeled probe for methylated and a JOE-labeled probe for unmethylated DNA in a single reaction on a Corbett Rotorgene 6000 (Corbett Life Sciences, Australia). Using serial dilutions of the controls, methylated RARB was identified at a limit of $1.625 \%$ in a background of unmethylated DNA.

\subsection{BRAF Mutation Analysis}

The activating mutation V600E of BRAF was detected using a molecular beacon set as previously described [27]. DNAs extracted from cell lines were used as controls for homozygous mutated (SKMEL28), heterozygous mutated (HT29) and homozygous wild type 
(SKMEL2). DNA extracts and cell lines were acquired from Cell Lines Service (CLS, Eppelheim, Germany). Detection was performed on an ABI 7500 Real-Time PCR (Applied Biosystems, USA).

\subsection{Statistic Analysis}

Statistical analysis of the results was performed using Fisher's exact test for $2 \times 2$ tables, $\chi^{2}$ for tables with more than two parameters, Student's t-test using twotailed paired analysis for age differences and spearman's for bivariate correlation analysis.

\section{Results}

\subsection{Histology Findings}

Out of 74 cases only 12 were finally histological negative for malignancy while 3 had a hyperlastic polyp present as displayed in Table 1. The remaining cases were diagnosed with some form of carcinoma. The most common type was endometrioid carcinoma, as expected, with the majority of them being of grade I or II. Fewer cases of high grade III endometrioid carcinomas were found, followed by mixed type carcinomas. Only few serous and clear cell carcinomas were found, while despite carcinosarcomas being rare, 4 cases were identified. The high percentage of carcinomas found is justified since all women who where included were symptomatic with a clinical diagnosis in favor of malignancy. As expected, type I cancers were more common in younger women with a mean age of 61 years of age vs. type II with 69 years of age $(p=0.016)$.

\subsection{Cytology Findings}

Cytology diagnosis was set on 73 cases with only 1 being inadequate $(1.3 \%)$ (Table 1). Results were compared with final histology diagnosis set in samples either from dilation and curettage (D\&C) or hysterectomy. Cytology was able to identify 49 of 59 carcinomas as carcinomas and 7 as hyperplasias with atypia. Only 3 carcinomas were misclassified; one serous as hyperplasia without atypia, one carcinosarcoma as negative and the single cytology inadequate sample was an endometrioid adenocarcinoma. All atypical or malignant cytology samples were also carcinomas in histology. Overall agreement was $95.9 \%$ with a kappa value of 0.883 when atypical or worse cytology was used.

\subsection{Topoisomerase IIa Expression}

Slides were examined by an experiences cytopathologist and were initially scored in three categories; negative, less than $10 \%$ of nuclei stained and over $10 \%$ of nuclei stained (Figure 1). However, initial analysis of the results revealed that only one negative case was positive for topoisomerase, so the categories were condensed in either negative or positive. Topoisomerase positivity was significantly higher in carcinomas as diagnosed with cytology $(p=0.002)$ or histology $(p=0.007)$ and correlated with BRAF mutations or presence of methylation in either CDKN2A or hMLH1.

\subsection{Promoter Methylation Status}

All samples yielded adequate DNA for analysis as reflected from results for the methylation and mutation analysis (Table 2). Promoter methylation (Figure 2) of CDKN2A and MGMT was identified in $21.6 \%$ of the samples and was found in samples diagnosed as carcinomas by either cytology $(\mathrm{p}=0.097)$ or histology $(\mathrm{p}=$ 0.031). CDKN2A promoter methylation correlated with topoisomerase IIa expression $(\mathrm{p}=0.014, \mathrm{CC} 0.384)$. The single inadequate for cytology sample that turned out to be an endometrioid carcinoma had methylation in the CDKN2A promoter.

Promoter methylation of hMLH1 was the most common finding with $41.9 \%$ of the samples having partial or full methylation of the gene's promoter. It was the only gene where methylation was statistically more common

Table 1. Comparison of cytology and histology.

\begin{tabular}{|c|c|c|c|c|c|c|c|c|}
\hline \multirow{2}{*}{ Cytology } & \multicolumn{7}{|c|}{ Histology } & \multirow{2}{*}{ Total } \\
\hline & Negative & Polyp & Endometrioid & Mixed & Clear cell & Serous & Carcinosarcoma & \\
\hline Negative & $11(91.7 \%)$ & $1(33.3 \%)$ & & & & & $1(25.0 \%)$ & $13(17.6 \%)$ \\
\hline Hyperplastic & $1(8.3 \%)$ & $2(66.7 \%)$ & & & & $1(25.0 \%)$ & & $4(5.4 \%)$ \\
\hline Atypical & & & $4(10.3 \%)$ & $1(11.1 \%)$ & $1(33.3 \%)$ & $1(25.0 \%)$ & & $7(9.5 \%)$ \\
\hline Carcinoma & & & $34(87.2 \%)$ & $8(88.9 \%)$ & $2(66.7 \%)$ & $2(50.0 \%)$ & $3(75.0 \%)$ & $49(66.2 \%)$ \\
\hline Total & 12 & 3 & 39 & 9 & 3 & 4 & 4 & 74 \\
\hline
\end{tabular}


Table 2. Immunocytochemical and molecular testing results.

\begin{tabular}{|c|c|c|c|c|c|c|c|c|c|}
\hline & & Topo IIa (\%) & $\begin{array}{c}\text { CDKN2A } \\
\text { Methylated (\%) }\end{array}$ & $\begin{array}{c}\text { hMLH1 } \\
\text { Methylated (\%) }\end{array}$ & $\begin{array}{c}\text { MGMT } \\
\text { Methylated (\%) }\end{array}$ & $\begin{array}{c}\text { RARB } \\
\text { Methylated (\%) }\end{array}$ & $\begin{array}{c}\text { Any Gene } \\
\text { Methylated (\%) }\end{array}$ & $\begin{array}{c}\text { BRAF } \\
\text { V600E (\%) }\end{array}$ & Total \\
\hline \multirow[t]{3}{*}{ Age } & Mean & 62.37 & 63.13 & $66.65^{*}$ & 61.88 & 67.2 & 64.3 & 57.6 & 62.9 \\
\hline & Inadequate & & $1(100 \%)$ & & & & $1(100 \%)$ & & $1(1.4 \%)$ \\
\hline & Negative & $1(7.7 \%)$ & & & & & & & $13(17.6 \%)$ \\
\hline \multirow[t]{6}{*}{ Cytology } & Hyperplastic & & & & & & & & $4(5.4 \%)$ \\
\hline & Atypical & $5(71.4 \%)$ & $3(42.9 \%)$ & $2(28.6 \%)$ & $2(28.6 \%)$ & $0(0 \%)$ & $5(71.4 \%)$ & $1(14.2 \%)$ & $7(9.5 \%)$ \\
\hline & Carcinoma & $21(42.9 \%)$ & $12(24.5 \%)$ & $29(59.2 \%)$ & $14(28.6 \%)$ & $5(10.2 \%)$ & $42(85.7 \%)$ & $4(8.1 \%)$ & $49(66.2 \%)$ \\
\hline & Negative & $1(8.3 \%)$ & & & & & & & $12(16.2 \%)$ \\
\hline & Polyp & & & & & & & & $3(4.1 \%)$ \\
\hline & Endometrioid & $19(48.7 \%)$ & $11(28.2 \%)$ & $23(59 \%)$ & $11(28.2 \%)$ & $2(5.1 \%)$ & $34(87.2 \%)$ & $5(12.8 \%)$ & $39(52.7 \%)$ \\
\hline \multirow[t]{5}{*}{ Histology } & Mixed & $3(33.3 \%)$ & $3(33.3 \%)$ & $6(66.7 \%)$ & $4(44.4 \%)$ & $2(22.2 \%)$ & $9(100 \%)$ & & $9(12.2 \%)$ \\
\hline & Clear cell & $1(33.3 \%)$ & $1(33.3 \%)$ & $1(33.3 \%)$ & $1(33.3 \%)$ & $0(0 \%)$ & $2(66.7 \%)$ & & $3(4.1 \%)$ \\
\hline & Serous & $2(50.0 \%)$ & $1(25 \%)$ & & & $1(25 \%)$ & $2(50 \%)$ & & $4(5.4 \%)$ \\
\hline & Carcinosarcoma & $1(25 \%)$ & & $1(25 \%)$ & & & $1(25 \%)$ & & $4(5.4 \%)$ \\
\hline & I & $4(26.7 \%)$ & $3(20 \%)$ & $7(46.7 \%)$ & $4(26.7 \%)$ & $1(6.7 \%)$ & $13(86.7 \%)$ & $1(6.7 \%)$ & $15(25 . \$ \%)$ \\
\hline \multirow[t]{3}{*}{ Grade } & II & $9(56.3 \%)$ & $3(18.8 \%)$ & $11(68.8 \%)$ & $3(18.8 \%)$ & & $13(81.3 \%)$ & $4(25 \%)$ & $16(27.1 \%)$ \\
\hline & III & $13(46.4 \%)$ & $10(35.7 \%)$ & $13(46.4 \%)$ & $9(32.1 \%)$ & $4(14.3 \%)$ & $22(78.6 \%)$ & & $28(52.5 \%)$ \\
\hline & I & $13(41.9 \%)$ & $6(19.4 \%)$ & $18(58.1 \%)$ & $7(22.6 \%)$ & $1(3.2 \%)$ & $26(83.9 \%)$ & $5(16.2 \%)$ & $31(52.5 \%)$ \\
\hline Type & II & $13(46.4 \%)$ & $10(35.7 \%)$ & $13(46.4 \%)$ & $9(32.1 \%)$ & $4(14.3 \%)$ & $22(78.6 \%)$ & & $28(47.5 \%)$ \\
\hline & otal (\%) & $26(35.1 \%)$ & $16(21.6 \%)$ & $31(41.9 \%)$ & $16(21.6 \%)$ & $5(6.8 \%)$ & $48(64.9 \%)$ & $5(6.8 \%)$ & 74 \\
\hline \multicolumn{2}{|c|}{ Total of carcinomas (\%) } & $25(42.4 \%)$ & $16(27.1 \%)$ & $31(52.5 \%)$ & $16(27.1 \%)$ & $5(8.4 \%)$ & $48(81.3 \%)$ & $5(8.4 \%)$ & 59 \\
\hline
\end{tabular}

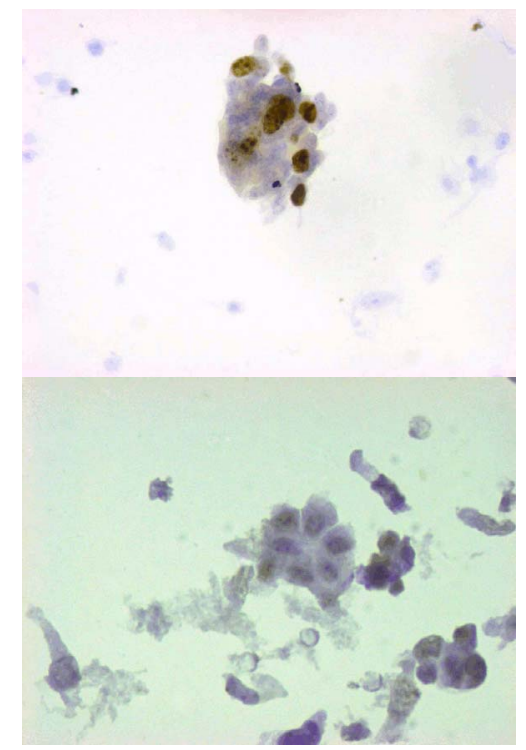

Figure 1. Immunocytochemistry for Topoisomare IIa expression. A negative sample (Bottom) and a strongly positive sample (Top) are displayed.

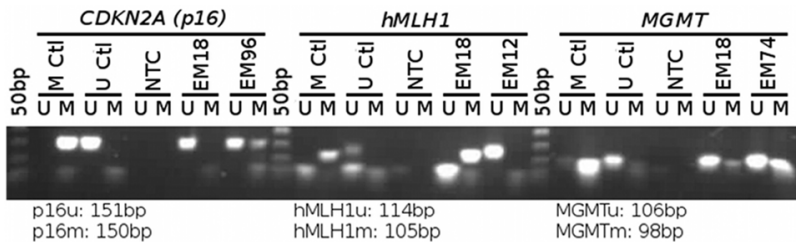

Figure 2. Methylation specific PCR for CDKN2A, hMLH1 and MGMT. Both controls, no template controls and clinical samples are shown.

in women of higher age $(\mathrm{p}=0.034)$, while methylation of hMLH1 was correlated with topoisomerase IIa expression ( $\mathrm{p}=0.022, \mathrm{CC}$ 0.267). Same as CDKN2A, hMLH1 methylation was more common in cancers $(\mathrm{p}<0.001)$.

Promoter methylation of RARB (Figure 3) was a rare event present only in 5 samples $(6.8 \%)$. Even though methylation was more common in type II cancers, due to the limited number of positive results, no statistical significance was identified. When combining all the above, at least one gene was methylated in 48 samples, out of which all were histological confirmed cancers, raising 
methylation at $64.9 \%$ of samples tested or $81.35 \%$ of cancer samples tested. While even combining all methylation results did not reach the statistical measures of diagnostic efficiency of cytology, the gap between them narrowed significantly (Table 3).

\subsection{BRAF Activating Mutation}

In total, 5 samples carried the V600E activating mutation (Figure 4). All were endometrioid carcinomas; one was a grade I adenocarcinomas and four were grade II. This
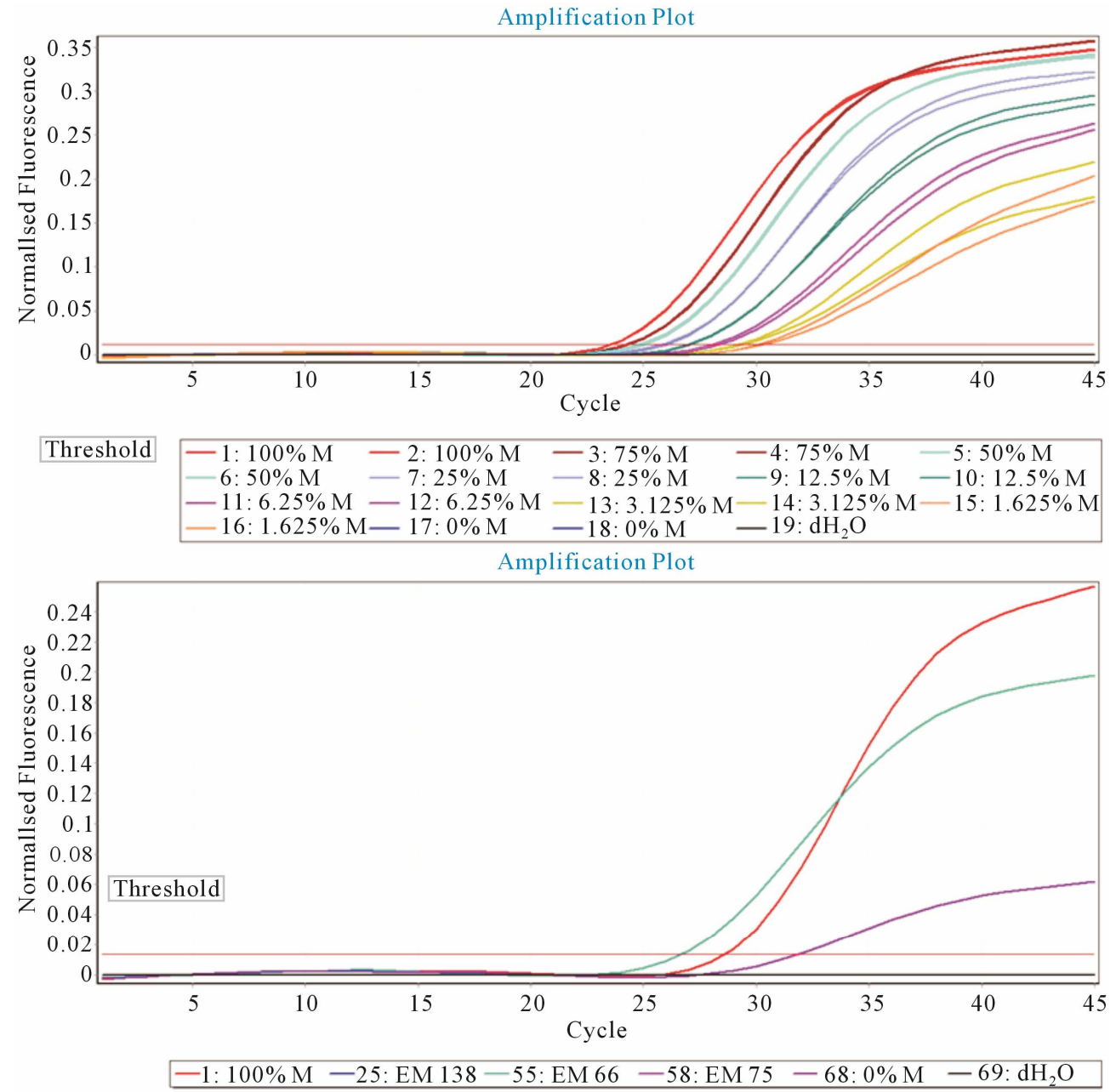

Figure 3. MethyLight detection of RARb. Serial dilutions were created from $100 \%$ methylated to $0 \%$ methylated DNA. Up to $1.6 \%$ of methylated DNA was detectable (Top). Clinical samples for the methylated reaction. Two highly positive samples and one low positive with fluorescence over the threshold.

Table 3. Efficacy of cytology and molecular testing.

\begin{tabular}{ccccccc}
\hline Histology endpoint: Carcinoma & Sensitivity (95CI) & Specificity (95CI) & PPV (95CI) & NPV (95CI) & p & Spearman Corr. \\
\hline Cytology: Atypical or worse & $94.9(85.5-98.9)$ & $100(78.2-100)$ & $100(93.6-100)$ & $83.3(58.6-96.4)$ & $<0.001$ & 0.889 \\
CDKN2A methylated & $27.1(16.3-40.2)$ & $100(78.2-100)$ & $100(79.4-100)$ & $25.8(15.2-39.0)$ & 0.023 & 0.265 \\
hMLH1 methylated & $52.5(39.1-65.7)$ & $100(78.2-100)$ & $100(88.8-100)$ & $34.8(21.0-50.9)$ & $<0.001$ & 0.428 \\
MGMT methylated & $27.1(16.3-40.2)$ & $100(78.2-100)$ & $100(79.4-100)$ & $25.8(15.2-39.0)$ & 0.023 & 0.265 \\
RARB methylated & $8.4(2.8-18.6)$ & $100(78.2-100)$ & $100(47.8-100)$ & $21.7(12.7-33.3)$ & 0.249 & 0.153 \\
Any gene methylated & $81.4(69.1-90.3)$ & $100(78.2-100)$ & $100(92.6-100)$ & $57.7(36.9-76.7)$ & $<0.001$ & 0.685 \\
Topoisomerase expression & $44.1(31.1-57.6)$ & $93.3(68.1-99.8)$ & $96.3(81.0-99.9)$ & $29.8(17.3-44.9)$ & 0.007 & 0.312 \\
BRAF V600E mutated & $8.4(2.8-18.6)$ & $100(78.2-100)$ & $100(47.8-100)$ & $21.7(12.7-33.3)$ & 0.249 & 0.153 \\
\hline
\end{tabular}



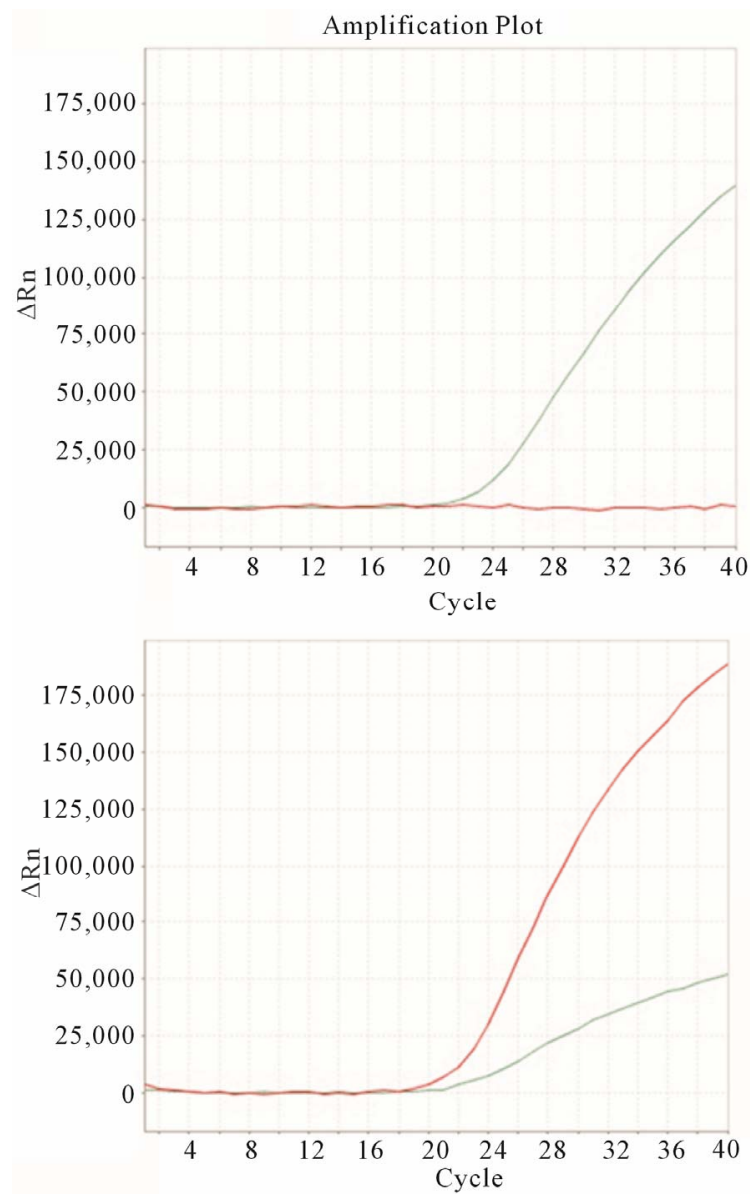

Figure 4. V600E detection using molecular beacons. Negative samples have positive signal for the JOE beacon (green line, top). Samples with a positive result in the FAM labeled beacon (red line, bottom) carry the mutation. Since samples also contain normal and inflammatory cells, V600E positive samples are usually also positive for the wild type probe.

reflected a percentage of $18.6 \%$ of type I carcinomas bearing the mutation, as opposed to $0 \%$ of type II ( $p=$ 0.056 ), with mutation being present more often in low grade carcinomas $(p=0.016)$. One sample had a cytology result of "hyperplasia with atypia, while the rest were diagnosed as carcinomas. BRAF mutation correlated with high topoisomerase IIa expression ( $\mathrm{p}<0.001$, $\mathrm{CC}=0.392)$ and presence of hMLH1 methylation $(\mathrm{p}=$ $0.026, \mathrm{CC}=0.259$ ). Even though mutations were found in younger women with a mean age of 57 years, due to the limited number of samples with mutation no statistic significance could be recognized.

\section{Discussion}

Even though cancer of the corpus uteri remains the most common gynecological related type of cancer in developed countries organised screening policies have not been set. There are several reasons for that. Firstly, endometrial carcinomas are presented with abnormal uterine bleeding and less than $1 \%$ are asymptomatic [14]. As a result, screening, especially in pre-menopausal women is not considered cost-effective [28]. On the other hand, in post-menaupasal women with abnormal thickening of the endometrium up to $42 \%$ of cancer cases were asymptomatic [22]. Furthermore, trans-vaginal ultrasound, which is the most common first line diagnostic test, has displayed low positive predictive value [15], especially in women that are under hormonal therapy. Finally, pippele biopsies only sample a limited region of the uteri $[15,16]$ and $\mathrm{D} \& \mathrm{C}$ requires patient admition and local anesthesia.

Cytology for the detection of endometrial malignancy has re-emerged, since more elaborate methods for sampling and storage of cells have been introduced. As a result, in many studies cytology has fewer inadequate samples than biopsies, while it remains less intervenetional and more tolerable $[19,29,30]$. In the limited number of the samples we examined a single sample which was inadequate, while all women found the sampling method very tolerable. Introduction of liquid based cytology has limited the factors reported to hamper safe cytology diagnosis [17], while many report extremely high diagnostic efficacy of liquid based cytology using current sampling and preparation methods [21,22]. In our samples, the only missed carcinomas were one that was inadequately sampled, a serous carcinoma and a single carcinosarcoma that are extremely rarely found. As a result high sensitivity, specificity and PPV were identified (Ta- ble 3). Even though the same diagnostic criteria of conventional cytology can be applied to liquid based cytology [20], hyperplasia with atypia was found to be the most controversial category among observers [31], while it has been propose that different commercial liquid based preparation methods may result in morphological alterations [32].

As a result we evaluated immunocytochemistry and molecular testing to identify whether they could be of ancillary use for or an alternate to cytology. Immunocytochemistry for hormone receptors and p53 expression in $\mathrm{LBC}$ has been previously used to profile tumors prior to hysterectomy [18], while mutation analysis in LBC has displayed similar results with FFPE tissue [33]. We found that adequate cells were available both for immunocytochemistry for topoisomerase IIa and for DNA extraction for mutation testing and methylation analysis in all samples.

In previous reports, increased expression of proliferation markers with concurrent deregulation of tumor suppressor genes has been found [34] and has been correlated with high grade aggressive carcinomas. However 
proliferating cells were also common in hyperplastic endometria without atypia. On the other hand we found a single case of a negative sample that expressed topoisomerase IIa, while the limited number of samples did not allow for an identification of a specific category that displays high topoisomerase IIa expression. Nevertheless, almost $50 \%$ of grade II and type II carcinomas have some expression, suggesting that targeted therapy against topoisomerase could have some beneficial use in endometrial carcinomas.

Methylation has been proposed as a screening or ancillary test for the detection of cancer in cervical samples, however with low sensitivity and or specificity $[24,35]$. Usually a combination of genes is used to further enhance test's results. We applied the same strategy, using a panel of four genes including tumor suppressors connected with either cell cycle regulation (CDKN2A, p16INK4A), or with epithelial differentiation (retinoic acid receptor RARB) and genes encoding DNA repair proteins as human MutL Homolog 1 (hMLH1) and O6Methylguanine DNA methyl-transferase (MGMT) was selected [23-26]. As previously found, hMLH1 methylation was the most common epigenetic effect identified. Loss of hMLH1 expression has been correlated with microsatellite instability (MSI) [36,37], and with higher grade and stage, when present along with specific mutations of PI3K or BRAF [38]. Even though we found that $80 \%$ of mutated BRAF had hMLH1 promoter methylation, the small number of BRAF mutated cases did not allow a statistical significance to be identified. Promoter methylation of either MGMT or CDKN2A was less often while they displayed a random profile with a small increase in type II carcinomas, as previously found in a limited number of endometrial carcinomas [36]. Others have reported significantly lower methylation of these genes using FFPE tissue stored for 10 years [39] or the COBRA technique [40]. RARb methylation was the rarest event observed only in 5 samples, out of which $80 \%$ were type II carcinomas. Usually methylation was exclusive for each gene with $66.7 \%$ of methylated samples having a single methylated gene, $25 \%$ two genes and $5.3 \%$ three genes. Including in the analysis all methylation events, all diagnostic efficacy statistics increased, though sensitivity and negative predictive value remained lower than cytology (Table 3).

Out of all three cancer cases negative for cytology only one had methylation in CDKN2A, while all the rest were negative for the biomarkers tested. As a result these tests did not offer substantial improvement. However, it would be interesting to study their positivity in larger scale studies that would include more cases of hyperplasia with and without atypia, since these represent the most controversial cytology categories.
Since LBC has been shown in several studies that it is a viable option for endometrial carcinoma detection [19-22,41] our findings were not surprising. However most studies published have a limited number of samples, while the one with over 900 cases has many inadequate biopsies [19]. Still the diagnostic efficacy of LBC we found was similar to previously published studies. The variance observed, especially in sensitivity, could be accounted to the significant number of type II carcinomas that were included in our study. The main goal of our study was analysis of complex molecular markers as ancillary to cytology. The excellent specificity of the limited number of markers we tested, even though it could not outperform cytology, suggests that in cases where cytology is equivocal molecular markers could be used, as others have proposed [33].

Distinct molecular profiles have been used to identify origin, grade and type of endometrial carcinomas [6], while distinct molecular events have been proposed for early detection while they are still in precursor forms $[9$, 10]. Apart from BRAF mutations that were exclusive in low grade endometrioid adenocarcinomas, none of the other biomarkers was specific with a statistical manner, partially due to the limited number of each of the subcategories of carcinomas we had available.

Novel targeted agents, either alone or in combination with cytotoxic agents and radiotherapy, may enhance treatment results or further widen treatment options of patients with endometrial carcinomas $[12,13,42]$. Our results indicate that apart from currently used regiments topoisomerase poisons [43], benefiting from topoisomerase IIa expression, alkylating agents, benefiting from MGMT methylation [36] and even retinoic acid [44,45] could be of potential use in a limited group of endometrial carcinomas.

\section{REFERENCES}

[1] A. Jemal, F. Bray, M. M. Center, J. Ferlay, E. Ward and D. Forman, "Global Cancer Statistics," CA: A Cancer Journal for Clinicians, Vol. 61, No. 2, 2011, pp. 69-90. doi: $10.3322 /$ caac. 20107

[2] F. Bray, I. Dos Santos Silva, H. Moller and E. Weiderpass, "Endometrial Cancer Incidence Trends in Europe: Underlying Determinants and Prospects for Prevention," Cancer Epidemiology, Biomarkers \& Prevention, Vol. 14, No. 5, 2005, pp. 1132-1142. doi:10.1158/1055-9965.EPI-04-0871

[3] A. S. Felix, L. S. Cook, M. M. Gaudet, T. E. Rohan, L. J. Schouten, V. W. Setiawan, et al., "The Etiology of Uterine Sarcomas: A Pooled Analysis of the Epidemiology of Endometrial Cancer Consortium," British Journal of Cancer, Vol. 108, No. 3, 2013, pp. 727-734. doi:10.1038/bjc. 2013.2

[4] J. V. Bokhman, "Two Pathogenetic Types of Endometrial 
Carcinoma," Gynecologic Oncology, Vol. 15, No. 1, 1983, pp. 10-17. doi:10.1016/0090-8258(83)90111-7

[5] J. Prat, "Prognostic Parameters of Endometrial Carcinoma," Human Pathology, Vol. 35, No. 6, 2004, pp. 649662. doi:10.1016/j.humpath.2004.02.007

[6] M. K. McConechy, J. Ding, M. C. Cheang, K. C. Wiegand, J. Senz, A. A. Tone, et al., "Use of Mutation Profiles to Refine the Classification of Endometrial Carcinomas," The Journal of Pathology, Vol. 228, No. 1, 2012, pp. 20-30.

[7] T. Okuda, A. Sekizawa, Y. Purwosunu, M. Nagatsuka, M. Morioka, M. Hayashi, et al., "Genetics of Endometrial Cancers," International Journal of Gynecology \& Obstetrics, Vol. 2010, 2010, Article ID: 984013.

[8] A. Yeramian, G. Moreno-Bueno, X. Dolcet, L. Catasus, M. Abal, E. Colas, et al., "Endometrial Carcinoma: Molecular Alterations Involved in Tumor Development and Progression," Oncogene, Vol. 32, No. 4, 2013, pp. 403413. doi:10.1038/onc.2012.76

[9] G. L. Mutter, T. A. Ince, J. P. Baak, G. A. Kust, X. P. Zhou and C. Eng, "Molecular Identification of Latent Precancers in Histologically Normal Endometrium," Cancer Research, Vol. 61, No. 11, 2001, pp. 4311-4314.

[10] L. Jia, Y. Liu, X. Yi, A. Miron, C. P. Crum, B. Kong, et al., "Endometrial Glandular Dysplasia with Frequent p53 Gene Mutation: A Genetic Evidence Supporting Its Precancer Nature for Endometrial Serous Carcinoma," Clinical Cancer Research, Vol. 14, No. 8, 2008, pp. 2263-2269. doi:10.1158/1078-0432.CCR-07-4837

[11] F. Amant, P. Moerman, P. Neven, D. Timmerman, E. Van Limbergen and I. Vergote, "Endometrial Cancer," Lancet, Vol. 366, No. 9484, 2005, pp. 491-505. doi:10.1016/S0140-6736(05)67063-8

[12] J. D. Wright, N. I. Barrena Medel, J. Sehouli, K. Fujiwara and T. J. Herzog, "Contemporary Management of Endometrial Cancer," Lancet, Vol. 379, No. 9823, 2012, pp. 1352-1360. doi:10.1016/S0140-6736(12)60442-5

[13] F. Zagouri, G. Bozas, E. Kafantari, M. Tsiatas, N. Nikitas, M. A. Dimopoulos, et al., "Endometrial Cancer: What Is New in Adjuvant and Molecularly Targeted Therapy?" International Journal of Gynecology \& Obstetrics, Vol. 2010, 2010, Article ID: 749579.

[14] L. G. Koss, K. Schreiber, S. G. Oberlander, M. Moukhtar, H. S. Levine and H. F. Moussouris, "Screening of Asymptomatic Women for Endometrial Cancer," CA: A Cancer Journal for Clinicians, Vol. 31, No. 5, 1981, pp. 300317. doi:10.3322/canjclin.31.5.300

[15] I. Symonds, "Ultrasound, Hysteroscopy and Endometrial Biopsy in the Investigation of Endometrial Cancer," Best Practice \& Research Clinical Obstetrics \& Gynaecology, Vol. 15, No. 3, 2001, pp. 381-391. doi:10.1053/beog.2000.0183

[16] M. M. Tahir, M. A. Bigrigg, J. J. Browning, S. T. Brookes and P. A. Smith, "A Randomised Controlled Trial Comparing Transvaginal Ultrasound, Outpatient Hysteroscopy and Endometrial Biopsy with Inpatient Hysteroscopy and Curettage," BJOG: An International Journal of Obstetrics \& Gynaecology, Vol. 106, No. 12, 1999, pp.
1259-1264. doi:10.1111/j.1471-0528.1999.tb08179.x

[17] S. B. Sams, H. S. Currens and S. S. Raab, "Liquid-Based Papanicolaou Tests in Endometrial Carcinoma Diagnosis. Performance, Error Root Cause Analysis, and Quality Improvement," American Journal of Clinical Pathology, Vol. 137, No. 2, 2012, pp. 248-254. doi:10.1309/AJCPLFBK1A2XJDQI

[18] J. Watanabe, Y. Nishimura, S. Tsunoda, M. Kawaguchi, I. Okayasu and H. Kuramoto, "Liquid-Based Preparation for Endometrial Cytology-Usefulness for Predicting the Prognosis of Endometrial Carcinoma Preoperatively," Cancer, Vol. 117, No. 4, 2009, pp. 254-263.

[19] A. M. Buccoliero, C. F. Gheri, F. Castiglione, F. Garbini, A. Barbetti, M. Fambrini, et al., "Liquid-Based Endometrial Cytology: Cyto-Histological Correlation in a Population of 917 Women," Cytopathology, Vol. 18, No. 4, 2007, pp. 241-249. doi:10.1111/j.1365-2303.2007.00463.x

[20] Y. Norimatsu, H. Kouda, T. K. Kobayashi, K. Shimizu, K. Yanoh, C. Tsukayama, et al., "Utility of Liquid-Based Cytology in Endometrial Pathology: Diagnosis of Endometrial Carcinoma," Cytopathology, Vol. 20, No. 6, 2009, pp. 395-402. doi:10.1111/j.1365-2303.2008.00589.x

[21] M. Papaefthimiou, H. Symiakaki, P. Mentzelopoulou, A. E. Giahnaki, Z. Voulgaris, E. Diakomanolis, et al., "The Role of Liquid-Based Cytology Associated with Curettage in the Investigation of Endometrial Lesions from Postmenopausal Women," Cytopathology, Vol. 16, No. 1, 2005, pp. 32-39. doi:10.1111/j.1365-2303.2004.00224.x

[22] C. Remondi, F. Sesti, E. Bonanno, A. Pietropolli and E. Piccione, "Diagnostic Accuracy of Liquid-Based Endometrial Cytology in the Evaluation of Endometrial Pathology in Postmenopausal Women," Cytopathology, 2012.

[23] E. J. Fox, D. T. Leahy, R. Geraghty, H. E. Mulcahy, D. Fennelly, J. M. Hyland, et al., "Mutually Exclusive Promoter Hypermethylation Patterns of hMLH1 and O6-Methylguanine DNA Methyltransferase in Colorectal Cancer," Journal of Molecular Diagnostics, Vol. 8, No. 1, 2006, pp. 68-75. doi:10.2353/jmoldx.2006.050084

[24] A. Spathis, E. Aga, M. Alepaki, A. Chranioti, C. Meristoudis, I. Panayiotides, et al., "Promoter Methylation of p16(INK4A), hMLH1, and MGMT in Liquid-Based Cervical Cytology Samples Compared with Clinicopathological Findings and HPV Presence," Infectious Diseases in Obstetrics and Gynecology, Vol. 2011, 2011, Article ID: 927861. doi/10.1155/2011/927861

[25] A. K. Virmani, C. Muller, A. Rathi, S. Zoechbauer-Mueller, M. Mathis and A. F. Gazdar, "Aberrant Methylation during Cervical Carcinogenesis," Clinical Cancer Research, Vol. 7, No. 3, 2001, pp. 584-589.

[26] T. Ivanova, A. Petrenko, T. Gritsko, S. Vinokourova, E. Eshilev, V. Kobzeva, et al., "Methylation and Silencing of the Retinoic Acid Receptor-Beta 2 Gene in Cervical Cancer," BMC Cancer, Vol. 2, 2002, p. 4.

[27] A. Spathis, J. Georgoulakis, P. Foukas, M. Kefala, K. Leventakos, A. Machairas, et al., "KRAS and BRAF Mutation Analysis from Liquid-Based Cytology Brush- 
ings of Colorectal Carcinoma in Comparison with Formalin-Fixed, Paraffin-Embedded Tissue," Anticancer Research, Vol. 30, No. 6, 2010, pp. 1969-1975.

[28] M. Kapali, N. P. Agaram, D. Dabbs, A. Kanbour, S. White and R. M. Austin, "Routine Endometrial Sampling of Asymptomatic Premenopausal Women Shedding Normal Endometrial Cells in Papanicolaou Tests Is Not Cost Effective," Cancer, Vol. 111, No. 1, 2007, pp. 26-33. doi:10.1002/cncr.22424

[29] B. R. Kipp, F. Medeiros, M. B. Campion, T. J. Distad, L. M. Peterson, G. L. Keeney, et al., "Direct Uterine Sampling with the Tao Brush Sampler Using a Liquid-Based Preparation Method for the Detection of Endometrial Cancer and Atypical Hyperplasia: A Feasibility Study," Cancer, Vol. 114, No. 4, 2008, pp. 228-235. doi:10.1002/cncr.23636

[30] A. R. Williams, S. Brechin, A. J. Porter, P. Warner and H. O. Critchley, "Factors Affecting Adequacy of Pipelle and Tao Brush Endometrial Sampling," BJOG, Vol. 115, No. 8, 2008, pp. 1028-1036. doi:10.1111/j.1471-0528.2008.01773.x

[31] M. Papaefthimiou, H. Symiakaki, P. Mentzelopoulou, A. Tsiveleka, A. Kyroudes, Z. Voulgaris, et al., "Study on the Morphology and Reproducibility of the Diagnosis of Endometrial Lesions Utilizing Liquid-Based Cytology," Cancer, Vol. 105, No. 2, 2005, pp. 56-64. doi:10.1002/cncr.21025

[32] Y. Norimatsu, S. Sakamoto, H. Ohsaki, S. Ozaki, T. Yokoyama, K. Shimizu, et al., "Cytologic Features of the Endometrial Adenocarcinoma: Comparison of ThinPrep and BD Surepath Preparations," Diagnostic Cytopathology, 2013.

[33] A. Di Lorito, S. Rosini, E. Falo, S. Gustapane, M. Gomes, J.L. Costa, et al., "Molecular Alterations in Endometrial Archived Liquid-Based Cytology," Diagnostic Cytopathology, 2012.

[34] A. Mourtzikou, K. Kosmas, A. Marouga, M. Stamouli, A. Pouliakis and P. Karakitsos, "The Use of an Immunocytochemical Double-Labeling Staining Can Display the Distribution of Bcl-2/Ki-67 Cells in Endometrial Adenocarcinomas as well as in Normal Endometrium," Clinical Laboratory, Vol. 58, No. 1-2, 2012, pp. 133-144.

[35] G. E. Kim, S. S. Kweon, J. S. Lee, J. H. Lee, J. H. Nam and C. Choi, "Quantitative Assessment of DNA Methylation for the Detection of Cervical and Endometrial Adenocarcinomas in Liquid-Based Cytology Specimens," Analytical and Quantitative Cytology and Histology, Vol. 34, No. 4, 2012, pp. 195-203.

[36] D. Furlan, I. Carnevali, B. Marcomini, R. Cerutti, E. Dainese, C. Capella, et al., "The High Frequency of de Novo Promoter Methylation in Synchronous Primary
Endometrial and Ovarian Carcinomas," Clinical Cancer Research, Vol. 12, No. 11, 2006, pp. 3329-3336.

[37] L. M. Peterson, B. R. Kipp, K. C. Halling, S. E. Kerr, D. I. Smith, T. J. Distad, et al., "Molecular Characterization of Endometrial Cancer: A Correlative Study Assessing Microsatellite Instability, MLH1 Hypermethylation, DNA Mismatch Repair Protein Expression, and PTEN, PIK3CA, KRAS, and BRAF Mutation Analysis," International Journal of Gynecological Pathology, Vol. 31, No. 3, 2012, pp. 195-205. doi:10.1097/PGP.0b013e318231fc51

[38] L. Catasus, A. Gallardo, M. Cuatrecasas and J. Prat, "PIK3CA Mutations in the Kinase Domain (Exon 20) of Uterine Endometrial Adenocarcinomas Are Associated with Adverse Prognostic Parameters," Modern Pathology, Vol. 21, No. 2, 2008, pp. 131-139.

[39] H. B. Salvesen, S. Das and L. A. Akslen, "Loss of Nuclear p16 Protein Expression Is Not Associated with Promoter Methylation But Defines a Subgroup of Aggressive Endometrial Carcinomas with Poor Prognosis," Clinical Cancer Research, Vol. 6, No. 1, 2000, pp. 153159.

[40] B. J. Rimel, P. Huettner, M. A. Powell, D. G. Mutch and P. J. Goodfellow, "Absence of MGMT Promoter Methylation in Endometrial Cancer," Gynecologic Oncology, Vol. 112, No. 1, 2009, pp. 224-228. doi:10.1016/j.ygyno.2008.08.038

[41] F. Garcia, B. Barker, J. Davis J, et al., "Thin Layer Cytology and Histopathology in the Evaluation of Abnormal Uterine Bleeding," The Journal of Reproductive Medicine, Vol. 48, No. 11, 2003, pp. 882-888

[42] N. Bansal, V. Yendluri and R. M. Wenham, "The Molecular Biology of Endometrial Cancers and the Implications for Pathogenesis, Classification, and Targeted Therapies," Cancer Control, Vol. 16, No. 1, 2009, pp. 8-13.

[43] S. J. Froelich-Ammon and N. Osheroff, "Topoisomerase Poisons: Harnessing the Dark Side of Enzyme Mechanism," The Journal of Biological Chemistry, Vol. 270, No. 37, 1995, pp. 21429-21432. doi:10.1074/jbc.270.37.21429

[44] K. Tanabe, H. Utsunomiya, M. Tamura, H. Niikura, T. Takano, K. Yoshinaga, et al., "Expression of Retinoic Acid Receptors in Human Endometrial Carcinoma," Cancer Science, Vol. 99, No. 2, 2008, pp. 267-271. doi:10.1111/j.1349-7006.2007.00684.x

[45] Y. H. Cheng, H. Utsunomiya, M. E. Pavone, P. Yin and S. E. Bulun, "Retinoic Acid Inhibits Endometrial Cancer Cell Growth via Multiple Genomic Mechanisms," Journal of Molecular Endocrinology, Vol. 46, No. 2, 2011, pp. 139-153. doi:10.1530/JME-10-0064 